\title{
Optimization of Photothermal Methods for Laser Hyperthermia of Malignant Cells Using Bioconjugates of Gold Nanoparticles
}

\author{
V. S. Gerasimov ${ }_{a}$, A. E. Ershov ${ }^{a, b, c}$, S. V. Karpov ${ }^{a, c, d}$, S. P. Polyutov ${ }^{a}$, and P. N. Semina ${ }^{c}$ \\ ${ }^{a}$ Siberian Federal University, Svobodnyi pr. 79, Krasnoyarsk, 660041 Russia \\ ${ }^{b}$ Institute of Computer Simulation, Siberian Branch, Russian Academy of Sciences, \\ Akademgorodok 50/44, Krasnoyarsk, 660036 Russia \\ ${ }^{c}$ Kirenskii Institute of Physics, Siberian Branch, Russian Academy of Sciences, \\ Akademgorodok 50/38, Krasnoyarsk, 660036 Russia \\ ${ }^{d}$ Reshetnev State Siberian State Aerospace University, pr. Gazety “Krasnoyarskii rabochii” 31, Krasnoyarsk, 660037 Russia \\ e-mail:karpov@iph.krasn.ru,valkrsk@gmail.com \\ Receqved 6.11.2015
}

\begin{abstract}
Selective action of laser radiation on membranes of malignant cells has been studied in different regimes using conjugates of gold nanoparticles with oligonucleotides by the example of DNA aptamers. Under the conditions of a contact between a bioconjugate and a cell surface and the development of substantial and rapidly relaxing temperature gradients near a nanoparticle, the membranes of malignant cells alone are efficiently damaged due to the local hyperthermia of a cellular membrane. It has been shown that the localization of the damaging action, which does not involve healthy cells, is realized, provided that pulsed, rather than continuous, laser radiation is used.
\end{abstract}

DOI: $10.1134 / \mathrm{S} 1061933 \mathrm{X} 16040050$

\section{INTRODUCTION}

Conjugates of plasmonic nanoparticles with biological macromolecules are important objects of interdisciplinary research, with studies in the fields of biomedicine and biotechnologies [1] having the greatest applied potential among them. The great interest in the investigation of these objects is due to the fact that they represent a technological platform for designing new-generation nanosized biosensors capable of detecting biomolecular interactions at the level of individual molecules. Biological complexes based on plasmonic nanoparticles are multifunctional and can simultaneously be used for diagnostics and therapy, as well as for medical visualization and monitoring of treating malignant neoplasms. Moreover, these bioconjugates are low toxicity, which is of special importance in the case of their application for early diagnostics and complex therapy of oncological diseases.

The photodynamic method for tumor treatment based on laser irradiation of affected tissues $[2,3]$ is a promising approach to solution of these problems. An alternative to the photodynamic method is a method that has been developed in the last decade for the selective thermal action by laser radiation on malignant-tumor-affected tissues labeled with plasmonresonant nanoparticles. This method has been named "plasmon-resonant photothermal therapy" (PRPTT) [4-6].

When using these methods, it is of importance to properly select the radiation wavelength that must coincide with both the minimum absorption by hemoglobin, which ensures the deepest penetration of the radiation, and the plasmon absorption band of nanoparticles. However, when biological tissues are irradiated with a laser beam in a real situation, it is also necessary to take into account the strong scattering caused by the optical heterogeneity of these tissues. Allowance for these factors is necessary to ensure that the radiation reaches the required depth [7] and control the temperature of the local heating of the biological tissues with plasmonic nanoparticles to provide irreversible damage of malignant cells. Hence, tuning of laser radiation parameters is an important condition for the photothermal treatment methods. The dependence of the spectral position of the plasmon absorption of gold nanoparticles on their sizes, shapes, and composition makes it possible to select a radiation wavelength that is optimum for the therapeutic effect in a range of 530-1100 $\mathrm{nm}[8,9]$.

When using tumor hyperthermia induced by the local superheating of oncological cells conjugated with nanoparticles, it is of special importance to ensure the selective action of laser radiation on an affected region without involving a healthy tissue. Note that the methods currently available for photodynamic treatment of tumors encounter complications and consequences that are destructive for healthy tissues. Therefore, the development of a very unintrusive and efficient method for treatment of malignant tumors is a major problem of oncology. 
Antibodies are prevalent among compounds that are capable of selective binding and, therefore, used in biomedical practice for the functionalization of nanoparticles. Their application in PRPTT has appeared to be efficient $[10,11]$. However, a rather large size of antibody molecules (of nearly $25-30 \mathrm{~nm}$ ) diminishes the efficacy of the photothermal action and its localization in a pulsed regime of irradiation because of a large gap between a nanoparticle and a cellular membrane surface.

In this connection, DNA aptamers are of independent interest owing to a number their advantages [1214]. Relatively small sizes (of $2-3 \mathrm{~nm}$ ) are among the most significant of them, because they make it possible to provide an optimum regime of heating oncological cells at a low intensity of laser pulses. Moreover, the contemporary methods for the synthesis of aptamers allow one to finely tune the properties of resulting conjugates, thus ensuring their selective binding with membrane surfaces of certain malignant cells.

The possibility of the targeted action of radiation on tumor cells alone without damaging healthy tissues is realized by introducing aptamer-nanoparticle bioconjugates into organism [12, 13]. Such bioconjugates, in the form of plasmonic nanoparticles with synthetic oligonucleotides-DNA aptamersadsorbed on their surface provide functionalization of these complexes and, as a consequence, precisely addressed delivery of the conjugates to a biological target. Therefore, the PRPTT process involving conjugates of gold nanoparticles with DNA aptamers is studied in this work.

The plasmonic nanoparticles most widely used for PRPTT include gold nanorods [15, 16], nanospheres $[9,17,18]$, and nanoshells [19-21]. Nanoparticles may be selectively attached to tumor cells via the functionalization of the nanoparticles with DNA aptamers in the cases of both passive [22] and active [12-14] delivery.

The aforementioned selective action is based on the ability of the aptamers to specifically bind complementary epitopes, i.e., regions of oncomarker proteins located on the membranes of malignant cells alone, while healthy cells do not interact with Au nanoparticles in this case. Gold nanoparticles bound to DNA aptamers absorb resonance laser radiation, are heated, and heat regions of a tumor via heat transfer. Elevation of their temperature to $42-43^{\circ} \mathrm{C}$ and above causes the apoptosis (controlled death) of malignant cells [2].

Review [5] reports results of a comparison of the photothermal actions of continuous and pulsed laser radiations on malignant cells under the conditions of the specific bonding of nanoparticles to the cells via antibodies. The approach based on the use of continuous laser radiation encounters a problem relevant to the low selectivity of the action on malignant cells, at which nanoparticles heated for a long time generate an isotropic temperature field with low radial gradients in an ambient medium. In this situation, both tumor cells directly adjacent to nanoparticles and healthy cells located at a rather large distance from the heat source undergo heating followed by necrosis. Thus, the longterm action of a continuous radiation leads to heating of the medium due to the heat transfer with the generation of a uniform temperature field, which covers the entire macroscopic region of the irradiated tissue. Under the conditions of a pulsed radiation in the absence of the specific binding, the main mechanism of cell damage is the mechanical action caused by the rapid appearance and disappearance of vapor cavities near nanoparticles. However, this mechanism requires a rather intense pulsed laser radiation [6, 23]. Our calculations (see Section 3) show that, when using aptamers (taking into account their small sizes) to bind nanoparticles to a cellular membrane, a thermal action sufficient for it to be damaged is reached at a temperature of the particle surface much lower than the threshold of water vaporization. This, in turn, makes it possible to use pulsed laser radiation with a significantly lower intensity.

In our previous works [24, 25], we studied the processes of the interaction of a pulsed laser radiation with spherical silver nanoparticles and their aggregates. Among other things, these studies considered taking into account the heating of an ambient medium by the example of water. It was found that the pulsed irradiation of such particles leads to the generation of rapidly relaxing thermal fields with high radial temperature gradients in the region directly adjacent to the particle surface.

Thus, there is reason to believe that the problem of the nonlocality of the hyperthermia of affected tissues may be solved by using a pulsed rather than a continuous laser radiation. In this case, a laser pulse acting on a nanoparticle for nearly $10^{-5}-10^{-8} \mathrm{~s}$ also heats the ambient medium, thus rapidly generating high radial temperature gradients, which relax in time periods of nearly $10^{-6}-10^{-7} \mathrm{~s}$. Therewith, only the cells closest to the nanoparticle appear to be located in the region heated to temperatures higher than that necessary for irreversible cell damage. However, traditional lasers that can be used in biomedicine generate nano-, pico, and femtosecond optical pulses with high peak intensities, which can induce irreversible thermal effects upon a nonselective thermolysis of cells. Therefore, separate studies are necessary to answer questions concerning the dependence of the rate of the apoptosis of malignant cells on the wavelength and intensity of laser radiation, as well as the pulse duration in a wide range of values, and their off-duty ratio under the conditions of a multipulse action. It has been experimentally shown [26] that selective laser hyperthermia of cells may be realized by treating a tumor with nanosecond laser pulses. However, our estimations have shown that, in this case, the nanoparticle temperature reaches several hundreds of degrees centigrade.

The goal of this work is to develop and optimize the photothermal methods for treatment of oncological 
diseases, these methods being based on the selective damage of malignant cellular membranes by subjecting them to laser irradiation at a wavelength of $532 \mathrm{~nm}$ under the conditions of the contact between the cells and a bioconjugate by the example of an aptamer-Au nanoparticle conjugate with regard to the characteristic sizes of the components.

\section{MODEL}

In this work, we consider the interaction of a gold nanoparticle, which is attached to a cellar membrane via an aptamer, with resonance pulsed laser radiation (in the form of a single pulse) at a wavelength of 532 $\mathrm{nm}$. The characteristic sizes of this system, including the membrane thickness (of nearly $5 \mathrm{~nm}$ [27]), are presented in Fig. 1.

Water is considered as a medium filling the interand intracellular spaces. The particle is irradiated by a plane electromagnetic wave,

$$
\mathbf{E}(\mathbf{r})=\mathbf{E}_{0} \exp (\mathrm{ik} \cdot \mathbf{r}),
$$

where $\mathbf{k}$ is the wave vector and $\mathbf{E}_{0}$ is the amplitude of the electric component of the electromagnetic field. The particle sizes are assumed to be much smaller than the radiation wavelength. The dipole moment induced on the particle is described by the following expression:

$$
\mathbf{d}=\varepsilon_{0} \alpha \mathbf{E}_{0},
$$

where $\mathbf{d}$ is the vector of the particle dipole moment, $\alpha$ is the dipole polarizability of the particle, and $\varepsilon_{0}$ is the electric constant.

In the general case, the particle polarizability with regard to the self-action [28] is determined as follows:

$$
\alpha=\left(\left(\alpha_{0}\right)^{-1}-\frac{2}{3} \mathrm{i}(R|\mathbf{k}|)^{3}\right)^{-1},
$$

where $\alpha_{0}$ is the particle polarizability with no regard to the effect of the self-action [28],

$$
\alpha_{0}=4 \pi R^{3} \frac{\varepsilon-\varepsilon_{\mathrm{m}}}{\varepsilon+2 \varepsilon_{\mathrm{m}}} .
$$

Here, $R$ is the particle radius, $\varepsilon$ is the dielectric permittivity of the particle material, and $\varepsilon_{\mathrm{m}}$ is the dielectric permittivity of the interparticle medium. The dependence of dielectric permittivity $\varepsilon$ on the particle radius is described by the following formula:

$$
\varepsilon=\varepsilon_{\mathrm{tab}}+\frac{\omega_{\mathrm{pl}}^{2}}{\omega\left(\omega-\mathrm{i} \Gamma_{0}\right)}-\frac{\omega_{\mathrm{pl}}^{2}}{\omega(\omega-\mathrm{i} \Gamma)},
$$

where $\varepsilon_{\mathrm{tab}}$ denotes the tabulated experimental values for a macroscopic sample [29]; $\Gamma_{0}$ is the electron relaxation constant for a macroscopic sample; $\omega_{\mathrm{pl}}$ is the plasma frequency of the particle material; and $\Gamma$ is the electron relaxation constant of a particle with regard its size dependence,

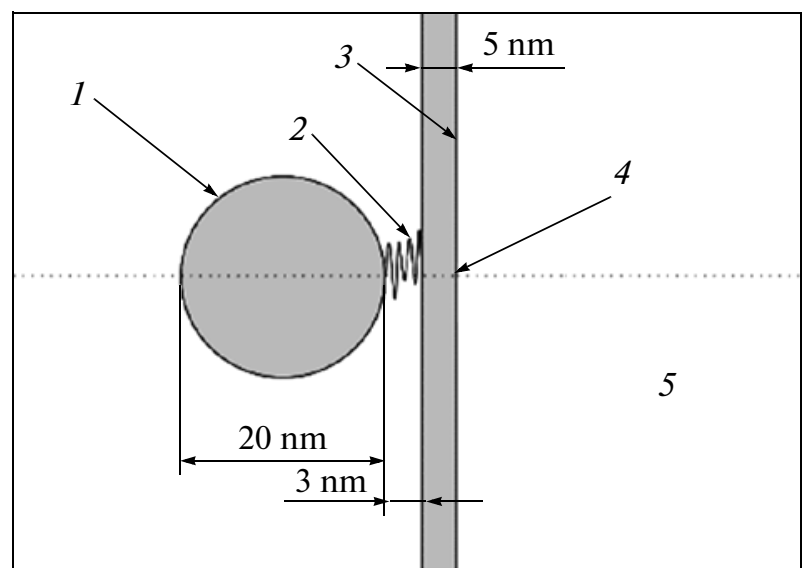

Fig. 1. Schematic representation of a nanoparticle and a cellular membrane: (1) Au nanoparticle, (2) aptamer, (3) cellular membrane, (4) point of temperature measurement, and (5) intracellular space.

$$
\Gamma=\Gamma_{0}+A \frac{V_{\mathrm{F}}}{R}
$$

where $V_{\mathrm{F}}$ is the Fermi electron velocity. Factor $A$ is taken to be equal to unity in the majority of model optical calculations. However, in the general case, relaxation processes at a particle surface depend on the state of the surface and a number of other factors.

Absorption cross-section $\sigma_{\mathrm{a}}$ is described by the following expressions [30]:

$$
\begin{gathered}
\sigma_{\mathrm{a}}=\frac{4 \pi}{k} y_{\mathrm{a}} \frac{|\mathbf{d}|^{2}}{\left|\mathbf{E}_{0}\right|^{2}}, \\
y_{\mathrm{a}}=\operatorname{Im}\left(\frac{1}{\alpha}\right)-\frac{2 k^{3}}{3} .
\end{gathered}
$$

When describing the thermodynamic processes relevant to heating of a particle material and an ambient medium at exposure times longer than $10^{-9} \mathrm{~s}$, the difference between the temperatures of the electronic and ionic components of the particle material may be ignored. This is because they are at thermodynamic equilibrium with each other, and the term "temperature" means the weight-average temperature of the ionic (crystal lattice) and electronic components of the particle. For the power of radiation absorbed by a particle, we have

$$
W=\sigma_{\mathrm{a}} I,
$$

where $I$ is laser radiation intensity.

The heating of an ambient medium by a particle may be described by the following equation for thermal conductivity:

$$
\frac{\partial T(\mathbf{r}, t)}{\partial t}-a(\mathbf{r}) \nabla^{2} T(\mathbf{r}, t)=f(\mathbf{r})
$$

where $a(\mathbf{r})$ is the coordinate-depending thermal diffusivity of the medium 


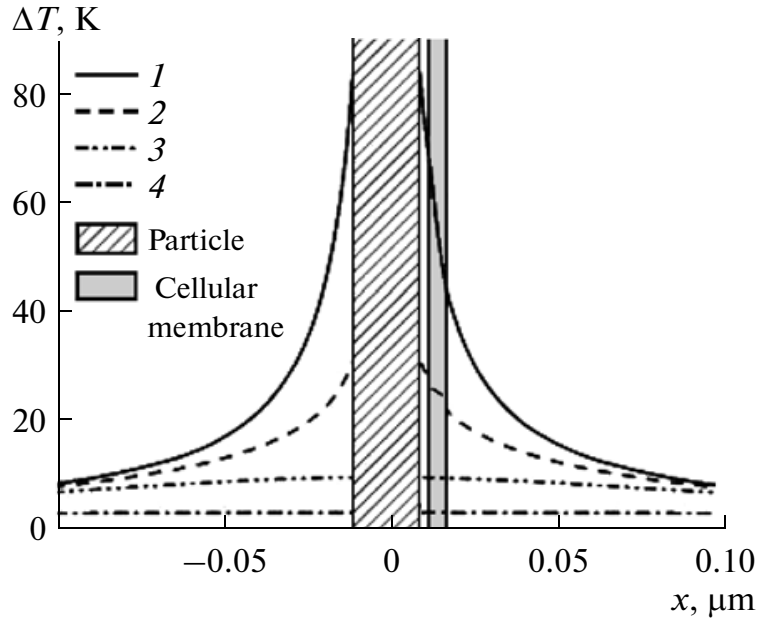

Fig. 2. Profiles of temperature increment $\Delta T$ near a particle being heated at different times elapsed after the pulse is switched on: (1) $10^{-6},(2) 1.008 \times 10^{-6}$, (3) $1.02 \times 10^{-6}$, and (4) $1.2 \times 10^{-6} \mathrm{~s}$. Pulse duration and wavelength are $\tau=$ $10^{-6} \mathrm{~s}$ and $\lambda=532 \mathrm{~nm}$, respectively.

$$
a(\mathbf{r})=\left\{\begin{array}{l}
a_{\mathrm{p}}, \text { at }|\mathbf{r}| \leq R, \\
a_{\mathrm{m}}, \text { at } D \leq x \leq D+h, \\
a_{\mathrm{int}}, \text { at } x>D+h, \\
a_{\mathrm{ex}}, \text { otherwise. }
\end{array}\right.
$$

Here, $\mathbf{r}=(x, y, z)$ is the radius vector for a point of the system within the space being heated, $a_{\mathrm{p}}$ is the thermal diffusivity of the particle material, $a_{\mathrm{m}}$ is the thermal diffusivity of a cellular membrane (the value for an adipose tissue of $9.8 \times 10^{-8} \mathrm{~m}^{2} \mathrm{~s}^{-1}$ [31] is used), $a_{\text {int }}$ is the thermal diffusivity of an intercellular liquid, $a_{\mathrm{ex}}$ is the thermal diffusivity of the intracellular substance (in this work, the difference between the thermal diffusivities of the media outside and inside a cell is ignored because of the high water content in them), $D$ is the distance between the particle center and the external surface of the membrane, and $h$ is the membrane thickness. The heat exchange between the particle and the ambient medium is described using the heat source function

$$
f(\mathbf{r})=\left\{\begin{array}{l}
\frac{W}{V}, \text { at }|\mathbf{r}| \leq R, \\
0, \text { otherwise, }
\end{array}\right.
$$

where $V$ is the particle volume.

In our problem, the following initial and boundary conditions have been specified: at initial time moment $t=0$, the temperature is taken to be the same throughout the system, $T(\mathbf{r}, t=0)=T_{0}$, and, at a distance equal to nearly half of the average interparticle distance (of about $1 \mu \mathrm{m}$ ) in the directions tangential to the membrane surface, symmetry condition $\partial T /\left.\partial r\right|_{|r|=1 \mu \mathrm{m}}=0$ is met.
Since the aptamer size is substantially smaller than the nanoparticle size, the thermodynamic model ignores the difference between the thermal diffusivities of the aptamer and the intercellular medium.

\section{RESULTS AND DISCUSSION}

Bioconjugates of spherical gold nanoparticles with a plasmon absorption band maximum at $520 \mathrm{~nm}$, which are most frequently applied in practice, were used in this work as model objects. Laser pulse duration is of importance for the selection of an optimum regime of irradiation, because, at an overly high radiation intensity, which is inherent in nanosecond pulses, the macroscopic processes of the local heating of tissues may be accompanied by photochemical decomposition and ionization of molecules with the formation of decomposition products.

\subsection{Action of Microsecond Pulses}

In spite of the high energy density, microsecond laser pulses were selected because of their relatively low intensity values, which make it possible to avoid occurrence of photochemical processes.

The pulse duration (from a few tenths of microsecond to one hundred of microseconds) and radiation intensity were varied in the calculations at a constant pulse energy density of $2.5 \mathrm{~J} / \mathrm{cm}^{2}$.

Figure 2 shows the spatial distributions of temperature in the medium surrounding a particle at the end of a laser pulse $10^{-6} \mathrm{~s}$ long (with a rectangular envelope curve) and in the course of particle cooling due to the heat exchange with the ambient medium. These distributions indicate that the thermal excitation relaxes very rapidly in the studied system. The maximum temperature increment decreases by three times over a time of nearly $10^{-8} \mathrm{~s}$, and thermodynamic equilibrium is established in the system in $10^{-7} \mathrm{~s}$. Thus, the local thermal action on the cellular membrane takes place only upon exposure to a laser pulse.

Figure 3 presents the temperature profiles for different laser pulse durations $\tau$ (Figs. $3 \mathrm{a}$ and $3 \mathrm{~b}$ differ in the scale alone). It can be seen that, at short pulse durations (less than $10^{-6} \mathrm{~s}$ ), a change in the temperature at a nanoparticle boundary is as high as $100 \mathrm{~K}$. At the same time, the temperature in the peripheral regions of the volume under consideration remains almost unchanged. In other words, only the local heating of a small region immediately adjacent to the particle takes place. However, at pulse durations of $10^{-5} \mathrm{~s}$ and longer, the thermal energy is distributed over the entire volume under consideration (Fig. 3b). Hence, in order to provide the conditions for the local heating, radiation with a pulse duration of shorter than $10^{-5}$ s should be used.

Figure 4 illustrates the time dependences of temperature increment $\Delta T_{\mathrm{m}}$ on the internal surface of the cellular membrane (see Fig. 1) for different laser pulse 

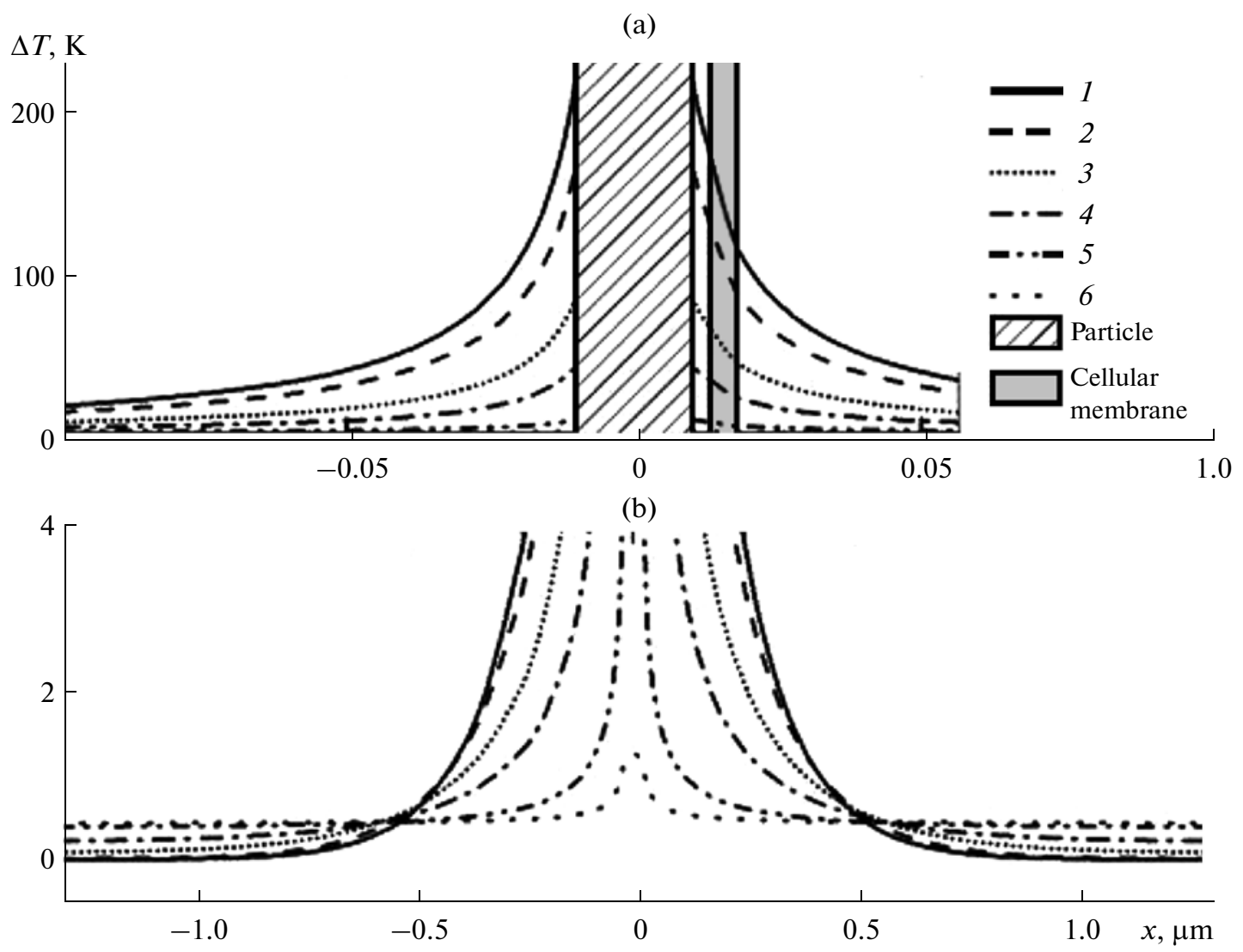

Fig. 3. Profiles of temperature increment $\Delta T$ near a particle at the end of a pulse in (a) large and (b) small scales at different pulse durations: $\tau=$ (1) 0.375 , (2) 0.5 , (3) 1 , (4) 2, (5) 10 , and (6) $100 \mu \mathrm{s} ; \lambda=532 \mathrm{~nm}$.

durations. It can be seen that the maximum membrane surface temperature, which is reached at the end of a pulse, rises with a decrease in the pulse duration. Moreover, the following tendency is observed: as the pulse duration increases, the heated region substantially enlarges. Hence, localized heating cannot be realized when continuous laser radiation is used.

The thermal damage of the cellular membrane is accompanied by dissolution of its lipids in the ambient aqueous electrolyte medium and conformational transformations of membrane proteins [32]. This process may be described by a simple kinetic equation of a first-order chemical reaction. The action inducing the damage of the cellular membrane may be described using the following formula, which is an analog of the Arrhenius equation [32]:

$$
\beta=\frac{d \Omega}{d t}=A_{\mathrm{r}} e^{-E / R T(t)},
$$

where parameter $A_{\mathrm{r}}$ characterizes the collision frequency of reacting molecules (at $T \rightarrow \infty$ ), $R$ is the gas constant, $T(t)$ is the time dependence of the cellular membrane temperature, $E$ is the activation energy [32], and $\Omega$ is a parameter characterizing the degree of the membrane damage. The degree of membrane damage may be determined by integrating Eq. (11) over the time of the thermal action. As applied to a group of cells or a tissue, the fraction of damaged cells may be determined using a simple kinetic model. Assume that cells occurring in initial state $\mathrm{O}$ have been subjected to a damaging thermal action, which transforms them into damaged state $\mathrm{B}$ at rate $\beta$ [32]:

$$
\begin{gathered}
\mathrm{O} \stackrel{\beta}{\longrightarrow} \mathrm{B}, \\
\frac{d N_{\mathrm{O}}}{d t}=-\beta(T(t)) N_{\mathrm{O}},
\end{gathered}
$$

where $N_{\mathrm{O}}$ is the number of cells in state $\mathrm{O}$. Therewith, the fraction of the damaged cells is

$$
k=1-\frac{N_{\mathrm{O}}(t)}{N_{\mathrm{O}}(0)}=1-e^{-\Omega(t)}
$$

In this expression, parameter $\Omega$ is obtained by solving Eq. (11) as follows:

$$
\Omega(t)=\int_{0}^{t} A_{\mathrm{r}} e^{-E / R T(t)} d t .
$$

Specific calculation of the efficiency of the thermal action was carried out at $A_{\mathrm{r}}=2.9 \times 10^{37} \mathrm{~s}^{-1}$ and $E=$ $244 \mathrm{~kJ} / \mathrm{mol}$, which are considered values typical of muscular tissue cells [33], with the damage of the cel- 


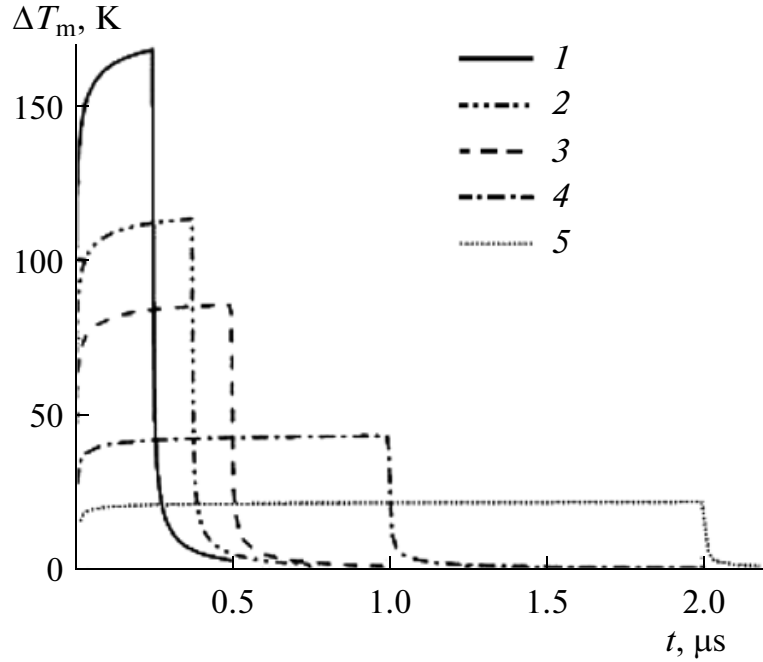

Fig. 4. Time dependences of maximum temperature increment $\Delta T_{m}$ at the internal surface of a cellular membrane at different laser pulse durations: $\tau=$ (1) 0.25 , (2) 0.375 , (3) 0.5 , (4) 1 , and (5) $2 \mu \mathrm{s} ; \lambda=532 \mathrm{~nm}$.

lular membrane being used as a criterion of cell apoptosis. Note that the cells of malignant formations are more sensitive to the thermal action [34]; therefore, the aforementioned values of parameters $A_{\mathrm{r}}$ and $E$ ensure the selection of conditions for the reliable damage of such cells. The table presents the fractions of the tissue cells damaged as a result of the thermal action illustrated in Fig. 4.

As can be seen from the table, the highest efficiency is inherent in laser radiation with a pulse duration of $0.4-0.25 \mu \mathrm{s}$.

\subsection{Peculiarities of the Action of Nanosecond Pulses}

Lasers with nanosecond pulse duration are most common and are frequently used in medical practice. Therefore, the study of the thermal action of radiation pulses as short as these is of independent interest. We have calculated the temperature fields and the kinetics of cellular membrane heating for a 20 -ns laser pulse. Figure 5 illustrates the evolution of the temperature profile after the end of the pulse and the time dependence of cell surface temperature increment $\Delta T_{\mathrm{s}}$. The

Fraction of damaged cells as depending on the duration of a laser pulse with a wavelength of $532 \mathrm{~nm}$

\begin{tabular}{c|c|c}
\hline \multicolumn{1}{c|}{$\tau, \mathrm{s}$} & $\Omega$ & $k$ \\
\hline $0.25 \times 10^{-6}$ & 2645 & 1 \\
$0.375 \times 10^{-6}$ & 1.6 & 0.798 \\
$0.5 \times 10^{-6}$ & 0.01795 & 0.018 \\
$1 \times 10^{-6}$ & $6.047 \times 10^{-6}$ & $6.047 \times 10^{-6}$ \\
$2 \times 10^{-6}$ & $6.34 \times 10^{-8}$ & $6.34 \times 10^{-8}$ \\
\hline
\end{tabular}

pulse energy density, which was selected in a manner such that the fraction of damaged cells in the tissue was almost equal to unity (at $\Omega=2.76$ ), appeared to be 88 $\mathrm{mJ} / \mathrm{cm}^{2}$, which is an order of magnitude lower than the value characteristic of microsecond radiation. Figure 5 shows that the temperature near the particle is higher than that for longer pulses because of a higher radiation intensity. In addition, the kinetics of variations in the temperature is distinguished by the following features: the temperature near the membrane decreases much more rapidly after the end of the pulse and continuously grows during the pulse action without passage to a stationary regime.

\section{CONCLUSIONS}

The data obtained confirm the following statements. The selectivity of the laser hyperthermia of malignant cells with no damage to healthy tissue is provided by the use of novel gold nanoparticle-DNA aptamer bioconjugates, in which aptamer sizes are specially selected. Therewith, the application of pulsed laser radiation under the conditions of nanoparticle contact with membranes of malignant cells alone via an aptamer makes a fundamental contribution to the localization of the action.

Pulses with both micro- and nanosecond durations are optimum from the point of view of the regime of thermal action on a cell membrane, provided that the radiation wavelength coincides with the position of the plasmon absorption band of nanoparticles.

Microsecond laser pulses are preferred for use in systems with a larger gap (several tens of nanometers) between a nanoparticle and a membrane.

The use of continuous laser radiation results in loss of the localization of the action on a cellular membrane because of the fast temperature relaxation of the region being heated and the formation of a wide uniform temperature field, which covers both malignant and healthy cells.

It has been established that the deleterious effect of the continuous laser radiation is aggravated at relatively large gaps (several tens and more of nanometers) between a nanoparticle and a cellular membrane surface, with such gaps additionally degrading the selectivity of the action on a cellular membrane and leading to heating of the entire region exposed to the laser radiation.

To increase the depth of laser radiation penetration into tissues, one should use biologically inert nanoparticles with plasmon resonance in a range of 600$800 \mathrm{~nm}$, in which hemoglobin is relatively transparent. Extended gold ellipsoids and nanorods, core/shell particles composed of cores with high refractive indices and gold shells are, in particular, among these nanoparticles. The results of studying systems based on such particles will be reported in a separate communication. 

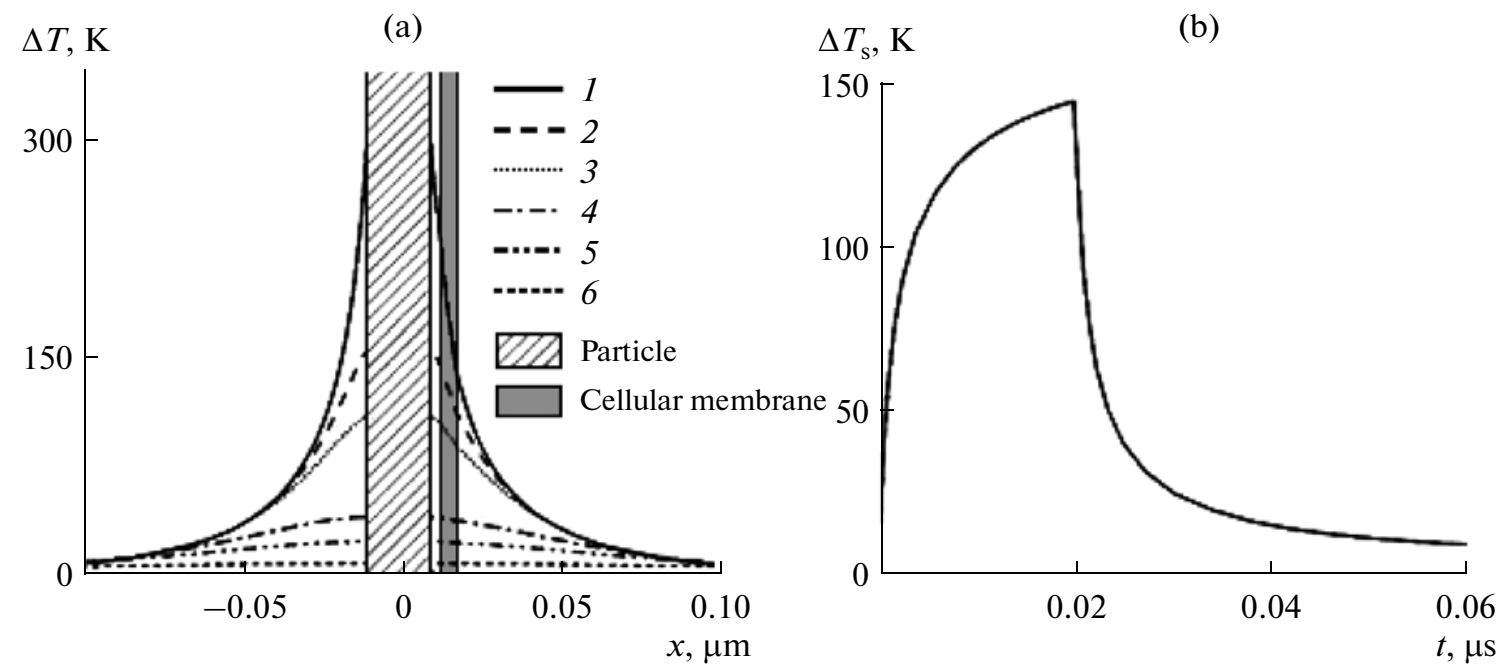

Fig. 5. Panel (a): profiles of temperature increment $\Delta T$ at different times $t$ elapsed after the end of a pulse: $t=(1) 2 \times 10^{-8}$, (2) 2.05 $\times 10^{-8},(3) 2.1 \times 10^{-8},(4) 2.5 @ \times 10^{-8}$, (5) $3.5 \times 10^{-8}$, and $(6) 5 \times 10^{-8}$ s and panel (b): time dependence of temperature increment $\mathrm{D} T_{S}$ on cellular membrane surface at $\tau=20 \mathrm{~ns}$ and $\lambda=532 \mathrm{~nm}$.

\section{ACKNOWLEDGMENTS}

This work was supported by the Ministry of Education and Science of the Russian Federation (contract no. 14.607.21.0104 RFMEFI60714X0104) (Section 3) and the State Assignment of the Ministry of Education and Science of the Russian Federation for Siberian Federal University (contract no. 1792) (Section 2). The numerical calculations were performed using the MVS-1000 M cluster at the Institute of Computer Simulation, Siberian Branch, Russian Academy of Sciences.

\section{REFERENCES}

1. Khlebtsov, N.G., Kvant. Elektron., 2008, vol. 38, p. 504.

2. Chumakov, D.S., Basko, M.V., Dikht, N.I., Bucharskaya, A.B., Rodionova, T.I., and Terentyuk, G.S., Saratovsk. Nauchno-Med. Zh., 2013, vol. 9, p. 700.

3. Habash, R.W.Y., Bansal, R., Krewski, D., and Alhafid, H.T., Crit. Rev. Biomed. Eng., 2007, p. 37.

4. Huang, X., Jain, P.K., El-Sayed, I.H., and ElSayed, M.A., Laser Med. Sci., 2008, vol. 23, p. 217.

5. Lapotko, D., Nanomedicine, 2009, vol. 4, p. 253.

6. Lukianova-Hleb, E., Hu, Y., Latterini, L., Tarpani, L., Lee, S., Drezek, R.A., Hafner, J.H., and Lapotko, D.O., ACS Nano, 2010, vol. 4, p. 2109.

7. Tuchin, V., Opticheskaya biomeditsinskaya diagnostika (Optical Biomedical Diagnostics), Moscow: Fizmatlit, 2007.

8. Huang, X. and El-Sayed, M.A., J. Adv. Res., 2010, vol. 1, p. 13.

9. Elbialy, N., Abdelhamid, M., and Youssef, T., J. Biomed. Nanotechnol., 2010, vol. 6, p. 687.

10. Dreaden, E.C., Mackey, M.A., Huang, X., Kang, B., and El-Sayed, M.A., Chem. Soc. Rev., 2011, vol. 40, p. 3391.
11. Dykman, L. and Khlebtsov, N., Chem. Soc. Rev., 2012, vol. 41, p. 2256.

12. Zhang, J., Liu, B., Liu, H., Zhang, X., and Tan, W., Nanomedicine, 2013, vol. 8, p. 983.

13. Reinemann, C. and Strehlitz, B., Swiss Medical Weekly, 2014, vol. 144, p. 13908.

14. Zamay, G.S., Kolovskaya, O.S., Zamay, T.N., Glazyrin, Y.E., Krat, A.V., Zubkova, O., Spivak, E., Wehbe, M., Gargaun, A., Muharemagic, D., Komarova, M., Grigorieva, V., Savchenko, A., Modestov, A.A., Berezovski, M.V., and Zamay, A.S., Mol. Ther., 2015, vol. 23, p. 1486.

15. Huang, X., El-Sayed, I.H., Qian, W., and El-Sayed, M.A., J. Am. Chem. Soc., 2006, vol. 128, p. 2115.

16. Von Maltzahn, G., Park, J.-H., Agrawal, A., Bandaru, N.K., Das, S.K., Sailor, M.J., and Bhatia, S.N., Cancer Res., 2009, vol. 69, p. 3892.

17. Afifi, M.M., El Sheikh, S.M., Abdelsalam, M.M., Ramadan, H., Omar, T.A., El Tantawi, M., AbdelRazek, K.M., and Mohamed, M., Oral. Surg., Oral. Med., Oral. Pathol., Oral. Radiol., 2013, vol. 115, p. 743.

18. Lukianova-Hleb, E.Y., Hanna, E.Y., Hafner, J.H., and Lapotko, D.O., Nanotechnology, 2010, vol. 21, p. 085102.

19. Hirsch, L.R., Stafford, R.J., Bankson, J.A., Sershen, S.R., Rivera, B., Price, R.E., Hazle, J.D., Halas, N.J., and West, J.L., Proc. Natl. Acad. Sci. USA, 2003, vol. 100, p. 13549.

20. Loo, C., Lin, A., Hirsch, L., Lee, M.H., Barton, J., Halas, N., West, J., and Drezek, R., Technol. Cancer Res. Treat., 2004, vol. 3, p. 33.

21. Maksimova, I.L., Akchurin, G.G., Khlebtsov, B.N., Terentyuk, G.S., Akchurin, G.G., Jr., Ermolaev, I.A., Skaptsov, A.A., Soboleva, E.P., Khlebtsov, N.G., and Tuchin, V.V., Med. Laser Appl., 2007, vol. 22, p. 199.

22. Greisch, K., J. Drug. Target., 2007, vol. 15, p. 457. 
23. Hleb, E.Y., Hafner, J.H., Myers, J.N., Hanna, E.Y., Rostro, B.C., Zhdanok, S.A., and Lapotko, D.O., Nanomedicine, 2008, vol. 3, p. 647.

24. Gavrilyuk, A.P. and Karpov, S.V., Appl. Phys. B, 2009, vol. 97, p. 163.

25. Ershov, A.E., Gavrilyuk, A.P., Karpov, S.V., and Semina, P.N., Appl. Phys. B, 2014, vol. 115, p. 547.

26. Pitsillides, C.M., Joe, E.K., Wei, X., Anderson, R.R., and Lin, C.P., Biophys. J., 2003, vol. 84, p. 4023.

27. Kozlov, M.M. and Chernomordik, L.V., Curr. Opin. Struct. Biol., 2015, vol. 33, p. 61.

28. Markel, V.A., Muratov, L.S., Stockman, M.I., and George, T.F., Phys. Rev. B: Condens. Matter, 1991, vol. 43 , p. 8183 .

29. Johnson, P.B. and Christy, R.W., Phys. Rev. B: Condens. Matter, 1972, vol. 6, p. 4370.

30. Markel, V.A., Shalaev, V.M., Stechel, E.B., Kim, W., and Armstrong, R.L., Phys. Rev. B: Condens. Matter, 1996, vol. 53, p. 2425.
31. Hasgall, P.A., Gennaro, D.F., Baumgartner, C., Neufeld, E., Gosselin, M.C., Payne, D., Klingenbock, A., and Kuster, N., ITIS Database for Thermal and Electromagnetic Parameters of Biological Tissues, 2015, Version 3.0. DOI: 10.13099/VIP21000-03-0.

32. Lee, R.C., Aarsvold, J.N., Chen, W., Astumian, R.D., Kelley, K.M., and Pliskin, N.H., Semin. Neurol., 1995, vol. 15, p. 367.

33. Cravalho, E.G., Toner, M., Taylor, D.C., and Lee, R.C., in Electrical Trauma: The Pathophysiology, Manifestations and Clinical Management, Lee, R.C., Cravalho, E.G., and Burke, J.F., Eds., Cambridge: Cambridge Univ. Press, 1992, p. 281.

34. Genfol'd, M.L. and Barchuk, A.S., Lazernaya selektivnaya gipertermiya $v$ lechenii zlokachestvennykh novoobrazovanii. Metodicheskie rekomendatsii (Laser Selective Hyperthermia in Therapy of Malignant Tumors. Methodical Recommemdations), St. Petersburg: GUN NII Onkologii im. N.N. Petrova, 2002.

Translated by A. Kirilin

SPELL: 1. OK 